\title{
ANALYSIS OF THE KENYAN INFLATION RATES: THE ARIMA APPROACH
}

\author{
Jane Mumbi Thuita \\ Department of Management Science, Chuka University \\ DOI: 10.46609/IJSSER.2021.v06i02.007 URL: https://doi.org/10.46609/IJSSER.2021.v06i02.007
}

\begin{abstract}
This paper modelled the Kenyan Inflation Rates using the ARIMA models and used the fitted model for forecasting. Annual time series data for the period January 2005-January 2020was used in the analysis, by using Box-Jenkins method and ARIMA model. Data were obtained from the World Bank website. The results showed that, there is a convergence between predictive values and actual values during the period of 2020. The results indicate that the inflation rates in Kenya will increase in the year 2021.
\end{abstract}

Keywords: ARIMA Model, Box-Jenkins, Forecasting, Inflation, Moving Average

\subsection{Introduction}

The primary focus of monetary policy in many countries has been the maintenance of a low and stable rate of aggregate price inflation as defined by such measures such as the consumer price index (CPI). The underlying justification is that inflation is costly because it undermines real, wealth that enhances the economic activity.

There are a number of approaches available for forecasting the inflation time series data. The most widely used approach is the Box-Jenkins method, which includes only the time series being forecast is known as univariate forecasting. Autoregressive integrated moving average (ARIMA) modelling is a specific subset of univariate modelling, in which a time series is expressed in terms of past values of itself (the autoregressive component) plus current and lagged values of a 'white noise' error term (the moving average component). This paper focused on ARIMA model for forecasting the inflation rates.

\subsection{Inflation in Kenya}

Inflation is generally referred to as the steady rise of prices of goods and services within the economy within a certain period. Such commodities as food, clothing, transport, and other 


\section{International Journal of Social Science and Economic Research}

ISSN: $2455-8834$

Volume:06, Issue:02 "February 2021"

consumer staples (FE Online, 2019). Inflation is seen as an indicative measure when there is a decrease in an individual's purchasing power per unit of a country's currency. Such a measure is presented in percentage.

In Kenya, inflation has caused more loss than gain. Such side effects of inflation include; income redistribution- happens when lower-income groups in the society are hit by the high domestic utilities like water and foods. This leads to regressive effects. Additionally, there are generally reduced real incomes in the country at large. Many employees tend to quit the jobs due to reduced wages which later causes the unemployment problem. Higher borrowing costs will result from the continued rise of goods and services. This happens when individuals seek loans and mortgages from lending institutions (Riley, 2020). These institutions will, in return, protect themselves from increased prices by hiking the interests of the loans. On investment, potential investors from foreign countries will not venture into the Kenya economy. Besides, Kenya's business competitiveness, Kenya's business competitiveness will make the product from the said country less favorable in the international market. Despite the adverse effects of inflation, there are potential beneficiaries from rising inflation. Debtors in case their interest rates are negative, which cause the real value of debt to fall. Subsequently, workers who have strong wage bargaining power will have strong wage protection hence not affected by rising inflation instead of workers from weak jobs without unions to protect them.

The inflation trend in Kenya is characterized by peaks and troughs, leading to inflation cycles lasting for several months. The strong inflation inertia is essential in understanding inflation dynamics in Kenya and has implications on whichever monetary policy framework. The central bank pursues to achieve its objective of stable and low inflation. For instance, the inflationtargeting framework implies that inflation is likely to persist in subsequent periods, implying missing inflation targets in subsequent periods. This persistence could be explained by structural rigidities in the economy, mainly emanating from the supply side. This would imply that addressing supply-side constraints can play an essential role in lowering inflation and minimizing deviations from the middle path. Additionally, the government stability on governance pertaining economy has influenced inflation in both negative and positive ways. Political instabilities in Kenya have seen the country surging in deep inflation, for instance, during 2007 post-election violence.

\subsection{Autoregressive Integrated Moving Average Arima) Models}

The autoregressive moving average (ARMA) processes are the most widely known and applied set of linear time series models. For the $\operatorname{ARMA}(\mathrm{p}, \mathrm{q})$ process, the observation $X_{t}$ is linearly related to the $\mathrm{p}$ most recent observations $\left(X_{t-1}, \ldots, X_{t-p}\right)$, q most recent forecast errors 
$\left(\varepsilon_{t-1}, \ldots, \varepsilon_{t-q}\right)$ and the current disturbance $\varepsilon_{t}$ by the relation:

$$
X_{t}=\sum_{i=1}^{p} \phi_{i} X_{t-i}+\sum_{i=1}^{q} \theta_{i} \varepsilon_{t-i}+\varepsilon_{t}, \varepsilon_{t} \sim W N\left(0, \sigma^{2}\right)
$$

where $\phi_{i}$ and $\theta_{i}$ are model parameters. The equation in 1 represents an ARMA (p,q) process. Alternatively, using the backshift operator, an ARMA (p,q) process is represented as $\Phi(B) w_{t}=\Theta(B) \varepsilon_{t}$, where B is a backshift operator such that $\mathrm{BX}_{\mathrm{t}}=\mathrm{X}_{\mathrm{t}-1},\left\{\varepsilon_{t}\right\}$ is a sequence of uncorrelated random variables with zero mean and variance $\sigma^{2}$.The polynomials:

$$
\begin{aligned}
& \Phi(B)=1+\phi_{1} B+\phi_{2} B^{2}+\ldots+\phi_{p} B^{p} \\
& \Theta(B)=1+\theta_{1} B+\theta_{2} B^{2}+\ldots+\theta_{q} B^{q}
\end{aligned}
$$

represent the autoregressive and the moving average operators of order $\mathrm{p}$ and $\mathrm{q}$ respectively. The coefficients in $\Phi(B)$ and $\Theta(B)$ represent some of the model parameters.

A special case of the $\operatorname{ARMA}(\mathrm{p}, \mathrm{q})$ process is known as the autoregressive process which date back to the work of Yule (1927) where he developed the first order autoregressive process (AR(1)) which is given by the relation $X_{t}=\phi X_{t-1}+\varepsilon_{t}$ where $\phi$ is a model parameter and $\varepsilon_{t} \sim W N\left(0, \sigma^{2}\right)$. In general, the $\mathrm{AR}(\mathrm{p})$ process is represented as

$$
X_{t}=\sum_{i=1}^{p} \phi_{i} X_{t-i}+\varepsilon_{t}
$$

where $\phi_{i}$ are constants and $\varepsilon_{t} \sim W N\left(0, \sigma^{2}\right)$

Another type of linear time series model is known as the moving average (MA) process which was developed by Slutzky (1937). The functional form for the first order moving average process (MA (1)) process is given by the equation $X_{t}=\theta \varepsilon_{t-1}+\varepsilon_{t}$, where $\theta$ is model parameter and $\varepsilon_{t} \sim W N\left(0, \sigma^{2}\right)$. The general representation of an MA(q) process is given as

$$
X_{t}=\sum_{i=1}^{q} \theta_{i} \varepsilon_{t-i}+\varepsilon_{t}
$$

where $\theta_{i}$ are model parameters and $\varepsilon_{t} \sim W N\left(0, \sigma^{2}\right)$. Here $\varepsilon_{t}$ is not observable. 
International Journal of Social Science and Economic Research

ISSN: 2455-8834

Volume:06, Issue:02 "February 2021"

When the stationarity condition is assumed, i.e. when the mean, variance and autocovariances of a process are invariant under time translations, then the process is modelled using the ARMA models. The ARMA models have been applied to modeling the UK interest rates.

The ARIMA model is usually characterized by three terms namely $(\mathrm{p}, \mathrm{d}, \mathrm{q}) \mathrm{p}$ is the order of the AR term, 1 is the order of the MA term while $q$ is the number of differencing required to make the series stationary.

\subsection{Methodology}

The ARIMA methods for forecasting time series was utilized for this research. Unlike other methods they do not assume knowledge of any underlying economic model or structural relationships. Here, assumed that past values of the series plus previous error terms contain information for the purposes of forecasting. The main advantage of ARIMA forecasting is that it requires data on the time series in question only. This is advantageous if one is forecasting a large number of time series and also avoids a problem that occurs sometimes with multivariate models.

\subsection{Assumptions of ARIMA Model}

- Data should be stationary - by stationary it means that the properties of the series doesn't depend on the time when it is captured. A white noise series and series with cyclic behavior can also be considered as stationary series.

- Data should be univariate - ARIMA works on a single variable. Auto-regression is all about regression with the past values.

Steps to be followed for ARIMA modeling:

- Exploratory analysis

- $\quad$ Fit the model

- Diagnostic measures The first step in time series data modeling using $\mathrm{R}$ is to convert the available data into time series data format.

\subsection{Results and Discussions}

In this section, we present the results of the analysis. Before performing any exploratory data analysis, we need to analyze the three components of the time series data. These include the trend which is defined as the long-term increase or decrease in the data is referred to as a trend. This gives the underlying pattern in the data over time. The second is the seasonal variation. When a series is influenced by seasonal factors like the quarter of the year, month or days of a week seasonality exists in the series. It is always of a fixed and known period. The cyclic 
variation occurs when the data exhibit rises and falls that are not of the fixed period we call it a cyclic pattern.

\section{Exploratory Analysis}

To $\mathrm{d}$ the exploratory data analysis, the autocorrelation analysis to examine serial dependence and therefore help to estimate which value in the past has a correlation with the current value. This helps in providing the order $\mathrm{p}, \mathrm{d}, \mathrm{q}$ that is useful in estimating the ARIMA models. The spectral analysis can also be used to examine cyclic behavior. This is carried out to describe how variation in a time series may be accounted for by cyclic components. This is also referred to as a Frequency Domain analysis. Using this, periodic components in a noisy environment can be separated out. Trend estimation and decomposition is necessary for the seasonal adjustment. It seeks to construct, from an observed time series, a number of component series(that could be used to reconstruct the original series) where each of these has a certain characteristic.

The observed time series is visualized in the following time plot.
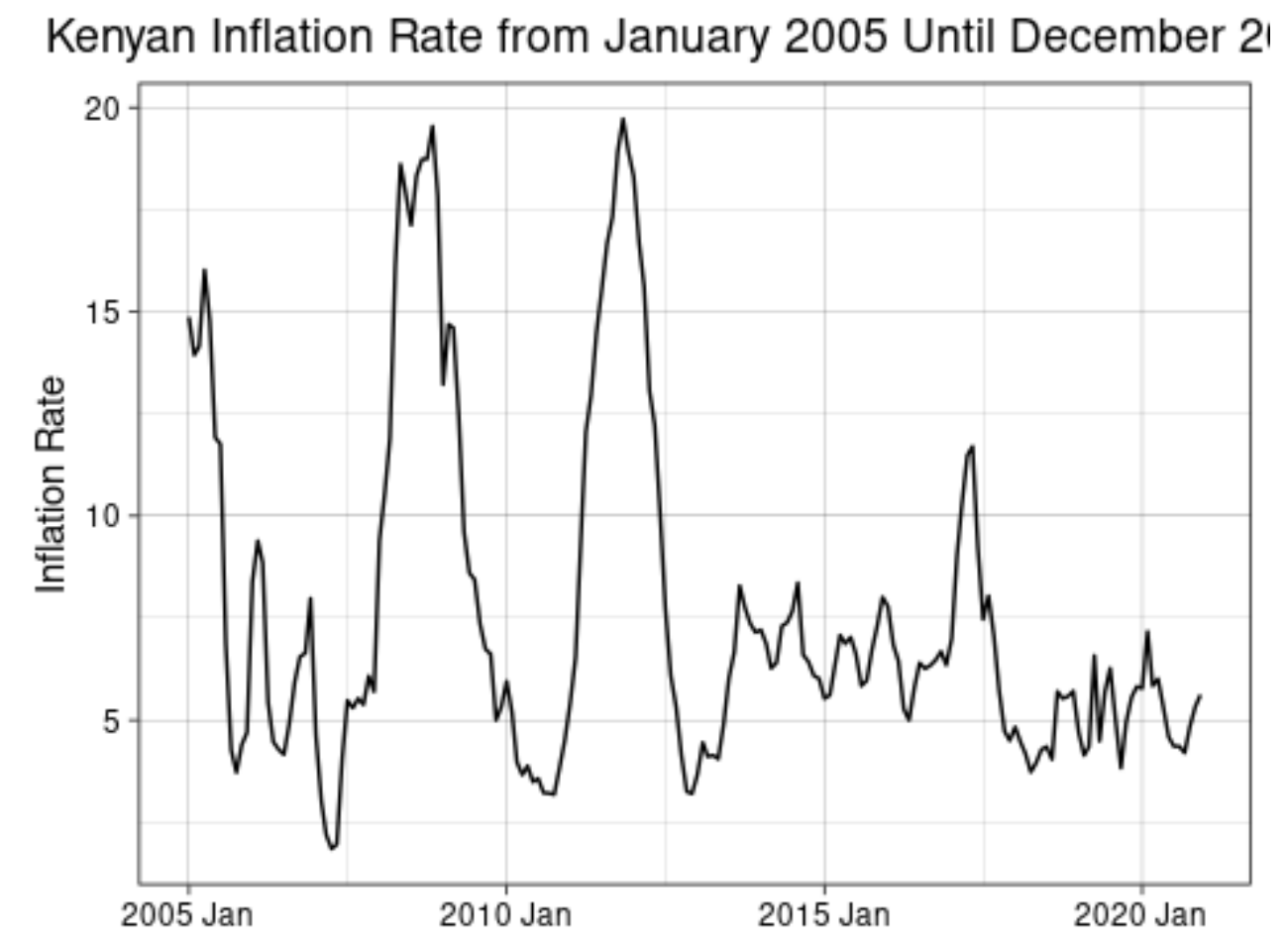

Figure 1:Kenyan Inflation Rate from January 2005 until December 2020

The additive decomposition of the inflation time series is presented in the Figure below. 


\section{Decomposition of additive time series}

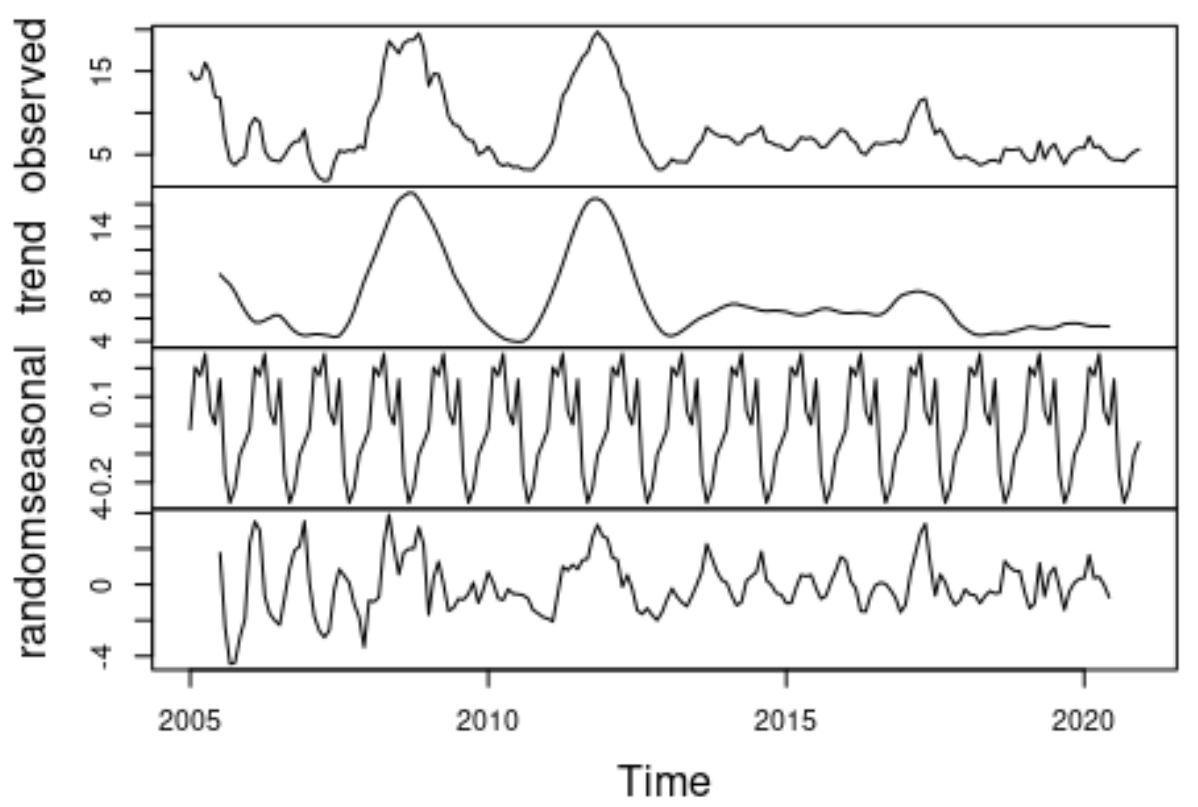

Figure 2: Decomposition of Additive Time Series

The four graphs reveal that data satisfies all the assumptions of ARIMA modeling. Next, we need to remove non-stationary part for ARIMA. For the sake of discussion here, we will remove the seasonal part of the data as well. The seasonal part can be removed from the analysis and added later, or it can be taken care of in the ARIMA model itself.

The Unit root test was used to find out whether the first difference or regression which should be used on the trending data to make it stationary. The Kwiatkowski-Phillips-Schmidt-Shin (KPSS) test was used to test for stationarity. A small p-values suggest differencing is required.

The raw data was transformed by finding the log difference 
International Journal of Social Science and Economic Research

Residuals from test regression of type: tau with 4 lags

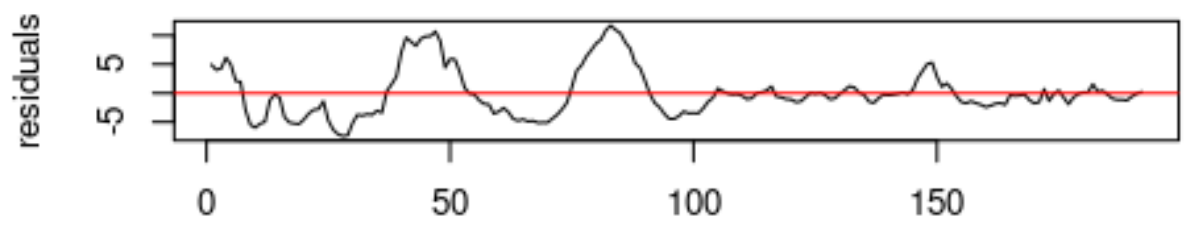

Autocorrelations of ResidualPartial Autocorrelations of Resid

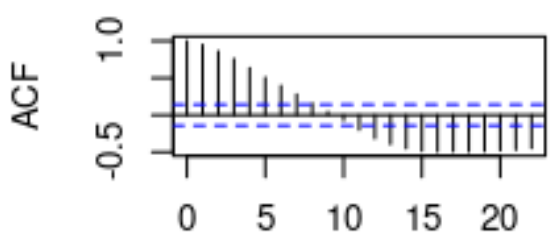

Lag

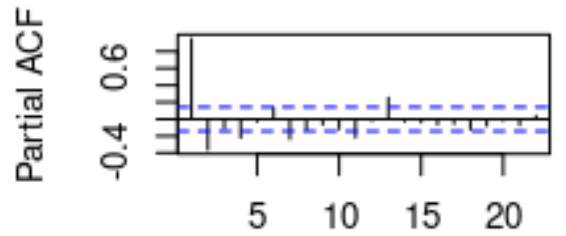

Lag

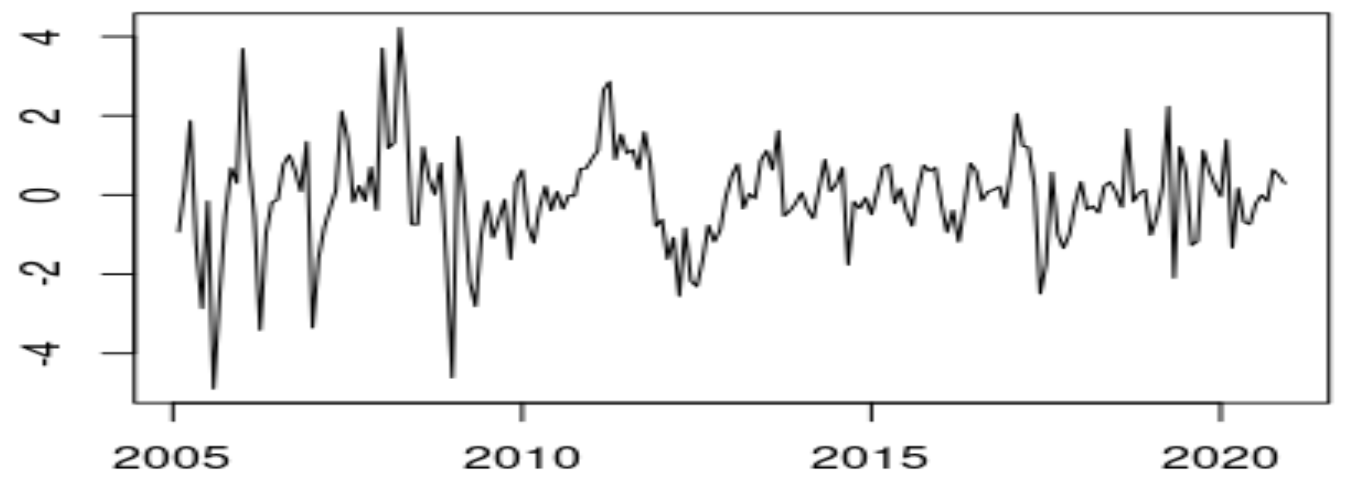

Time

Figure 3:Raw Data Transformed by Finding the Log Difference

To remove seasonality from the data, the seasonal component was subtracted from the original series and then differenced to make it stationary. 


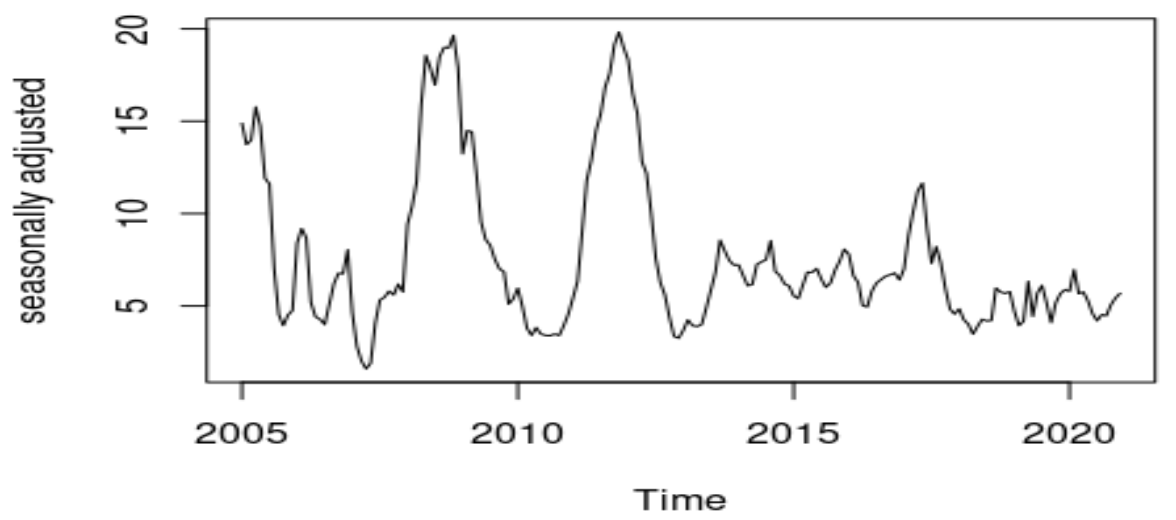

\section{Model Fitting}

We consider the ARIMA class of models to fit to the data. There are 2 models that we will consider for this case, there are Auto Regression (AR) model and also the Moving Average (MA) model. Before we use the model, we have to identify which model that fits on the data based on its autocorrelation and which lag suits the model. The Auto Correlation Function (ACF) plot and also Partial Auto Correlation Function (PACF) are used for order determination for these classes of models. See Figures below.

Usually, for AR(p) will fit the model if the ACF plot tails off and PACF plot cuts off to nearly 0 after lag $\mathrm{p}$. Then, the MA(q) model will fit if the ACF plot cuts off to nearly 0 after lag $\mathrm{Q}$ and the PACF plot tails off. If both plots are tailing off, then we can use the $\operatorname{ARMA}(p, q)$ model to it. Based on that information, the ACF plot decreases gradually (tail off) and the PACF plot decreases to nearly 0 after lag- 1 . Therefore, for this model, we will use the ARMA(1,1) model for the next step. 
International Journal of Social Science and Economic Research

ISSN: 2455-8834

Volume:06, Issue:02 "February 2021"
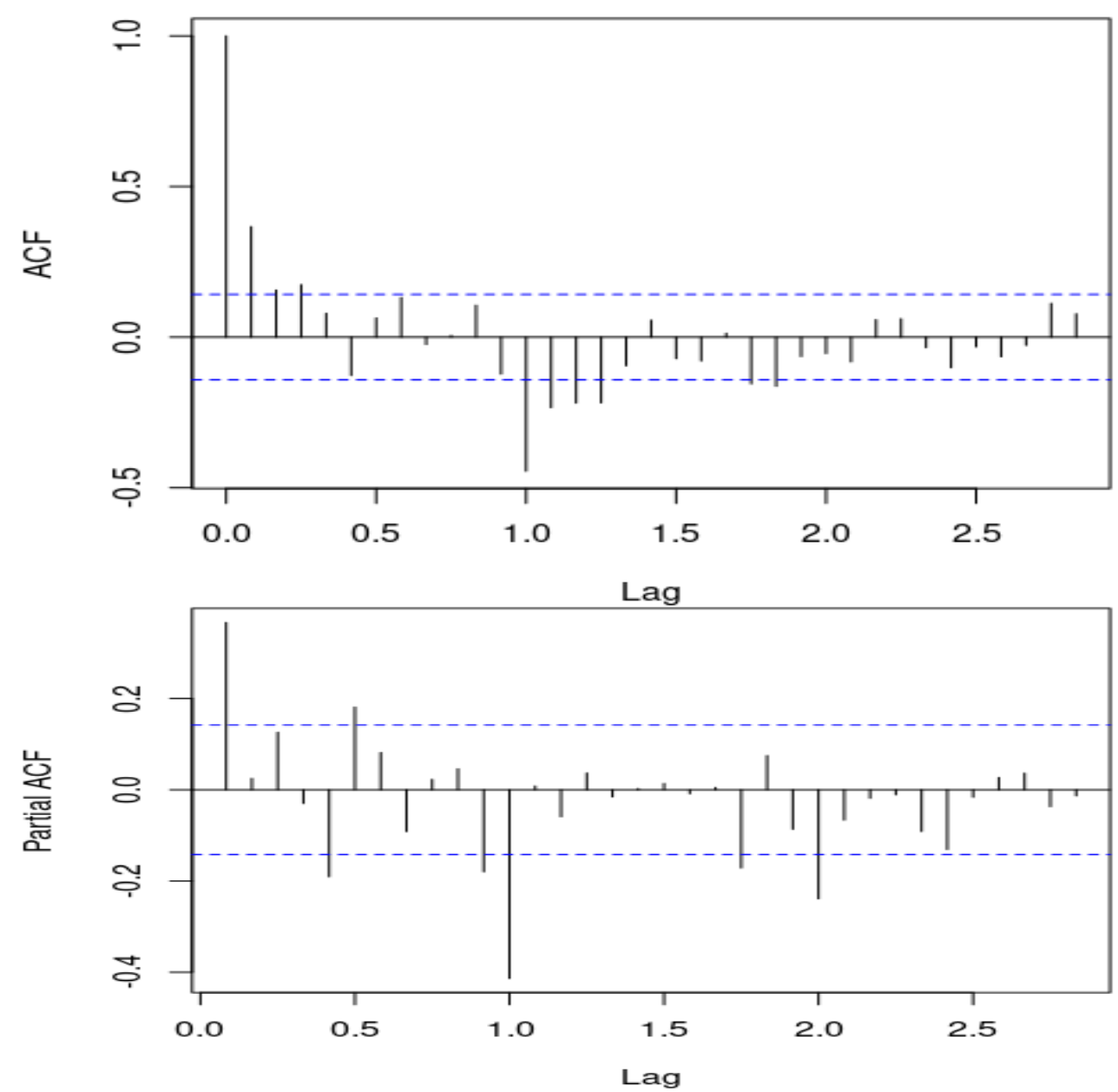

The fitted model is given by

Table 1: Fitted Model

\begin{tabular}{|l|l|l|l|l|}
\hline Parameter & Estimate & Std. Error & $\mathrm{z}$ value & $\operatorname{Pr}(>|\mathrm{z}|)$ \\
\hline ar1 & 0.548 & 0.180098 & 3.0432 & $0.002341^{* *}$ \\
\hline ma1 & -0.226082 & 0.212789 & -1.0625 & 0.288021 \\
\hline sar1 & -0.507760 & 0.067239 & -7.5516 & $4.299 \mathrm{e}-14$ *** \\
\hline
\end{tabular}

Signif.codes: 0 '***' $0.001^{\prime * *} 0.01^{\prime *} 0.05$ '.' $0.1^{\prime \prime} 1$

\section{Model Diagnosis}

Model diagnosis was carried out using Akaike's Information Criterion (AIC) for a set of models and investigate the models with lowest AIC values and Schwarz Bayesian Information Criterion (SBC). The model with the lowest value of the two measures was considered the best. 
Box-Ljung test is useful for testing the independence at all lags up to the one specified. Instead of testing randomness at each distinct lag, it tests the "overall" randomness based on a number of lags, and is therefore a portmanteau test. It is applied to the residuals of a fitted ARIMA model, not the original series, and here, the hypothesis actually being tested is that the residuals from the ARIMA model have no autocorrelation.
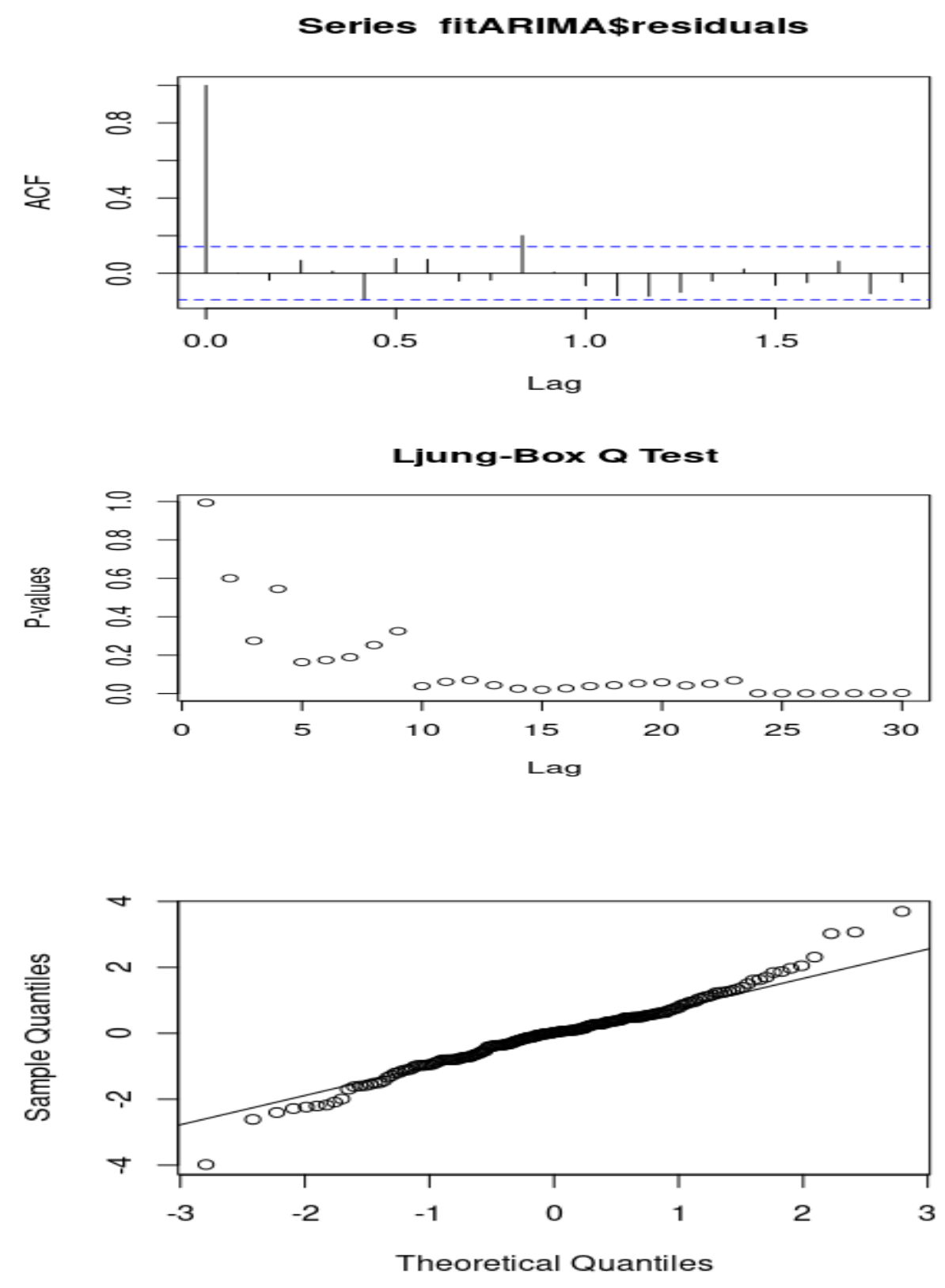


\section{Forecasting using an ARIMA model}

The parameters of that ARIMA model were used as a predictive model for making forecasts for future values of the time series once the best-suited model is selected for time series data.

The graph below shows the forecasted values.

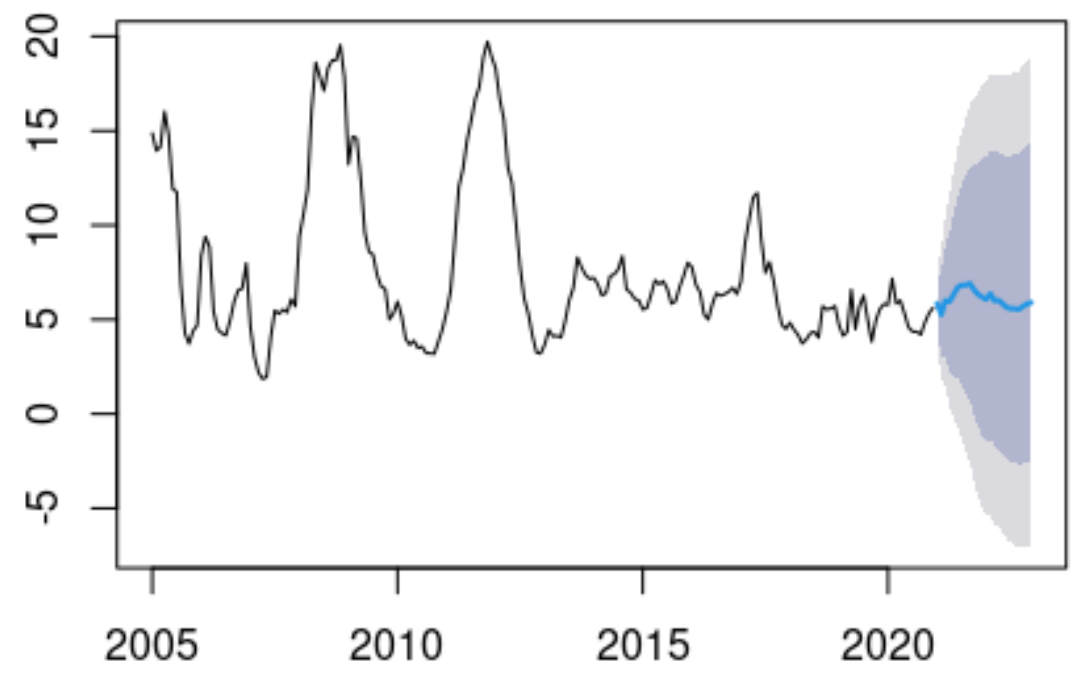

The d-value affects the prediction intervals, theta is, the prediction intervals increases in size with higher values of ' $d$ '. The standard long-term forecast standard deviation tended to the standard deviation of the historical data.

From the obtained results, the forecasted inflation for the next five months are $5.850,5.261,5.99,5.948,6.312$ This shows that the inflation is expected to rise and this will affact the consumers ability to purchase resulting in slowing down of the economy.

\subsection{Conclusions and Recommendations}

The study applied the ARIMA class of models in modelling the inflation rates for Kenya. The results showed that the models were suited for the analysis. The results further reveals that the inflation forecast expected to go higher as the year progresses. This raises concerns for the economic growth of Kenya. The government should put mitigation measures that would address the inherent rise in inflation rate in Kenya. 
International Journal of Social Science and Economic Research

ISSN: 2455-8834

Volume:06, Issue:02 "February 2021"

\section{References}

[1] FE Online. (2019, September 23). What is inflation? The Financial Express. Retrieved February 25, 2021, from https://www.financialexpress.com/what-is/inflation-meaning/1618981/

[2] Slustzky, H.(1937). The summation of random causes as the source of cyclic process. Econometrica.5, 105-146.

[3] Riley, G. (2020). Inflation - Consequences of inflation | Economics | tutor $2 u$. tutor2u. Retrieved February 25, 2021, from https://www.tutor2u.net/economics/reference/inflationconsequences-of-inflation 